\begin{tabular}{cccc}
\hline & $\begin{array}{c}\text { International Journal of Health Services } \\
\text { Research and Policy } \\
\text { www.dergipark.org.tr/ijhsrp }\end{array}$ \\
$\begin{array}{c}\text { INTEREG } \\
\text { ENGINEERING } \\
\text { SCIENCE AND } \\
\text { EDUCATION GROUP }\end{array}$ & e-ISSN: $2602-3482 \quad$ DOI:10.23884/ijhsrp.2019.4.3.05 & IJHSRP \\
\hline Research Article & &
\end{tabular}

\title{
THE VALUE OF DIFFERENT TUBERCULOSIS (TB) CASE DETECTION STRATEGIES IN CHILDHOOD TB CONTROL IN A HIGH-TB BURDEN POPULATION DENSE AREA, WESTERN KENYA, 2012-2015: A CLUSTER RANDOMIZED TRIAL
}

\author{
${ }^{*}$ Barbara K. Burmen ${ }^{1}$ iD, Timothy M. Malika ${ }^{2}$ iD \\ ${ }^{1}$ Kenya Medical Research Institute, Center for Global Health Research, P.o. Box 1578-40100, \\ Kisumu, Kenya \\ ${ }^{2}$ Division of Leprosy, Tuberculosis and Lung Disease, P.O. Box 20781 - 00202 Nairobi, \\ Kenya \\ *Corresponding author: drburmen@ gmail.com
}

\begin{abstract}
Contact investigation is recommended for close contacts of tuberculosis (TB) patients to identify undiagnosed cases of active and latent $T B$ to initiate them on curative and preventive therapy respectively. However, contact invitation is conducted in Kenya and therefore the value of TB contact investigation in childhood TB control is unknown. To compare the yield of contact investigation (intervention arm) to contact invitation (control arm) in contributing to childhood TB control, a clusterrandomized trial was conducted in Kisumu County between 2014 and 2015 a period prior to the implementation of standardized contact investigation. This was done to compare TB cases diagnosed and children receiving Isoniazid Preventive Therapy (IPT) in the pre-intervention (2012-2013) and intervention (2014-2015) years, and during in the intervening years using a minimum sample size of 15 per arm. Of 77 facilities identified for the study, 65 facilities were randomized to a contact screening strategy; a TB contact investigation strategy in isolation ( $n=4)$, in combination with health facility screening $(n=19)$, or in combination with both enhanced facility screening and mobile units $(n=31)$ with the remainder, $(n=11)$ randomized to the standard approach i.e. TB contact invitation. Facilities distribution did not differ by category of services or patient type. In the pre-intervention and postintervention years, the number of TB cases diagnosed in children increased by 20 (75\% from intervention arm). During the intervening years, TB cases decreased by 17 (29\% from intervention arm); the intervention arm contributed to $100 \%$ and $75 \%$ of the children put on IPT whose implementation had just begun. Contact investigation enhanced childhood TB control in comparison to routine approaches. Critical support ought to be availed to the TB screening cascade to facilitate contact investigation and IPT implementation as well as ingrain contact investigation within existing community health systems.
\end{abstract}

Keywords: Tuberculosis case detection strategies, contact investigation strategies, enhanced facilitybased screening, mobile units, childhood TB, high-TB burden, cluster-randomized trial, Kenya,

Received: May 21, 2019

Accepted: September 9, 2019

\section{Introduction}

Value in health care has been defined as health outcomes per dollar spent [1]. A valuable health care program is one that improves health care outcomes at a reasonable cost or achieves the most improvement with the available resources. This is because not all programs have a positive return on investment e.g. prevention programs across broad populations usually have low yield but result in a decrease in morbidity and mortality [2]. The value should always be defined around results i.e. the health outcomes achieved which are usually are disease-specific and multidimensional [1]. 
'Disease control' is defined as a decrease in the incidence, prevalence, morbidity, and mortality from the disease [3]. To reduce Tuberculosis (TB) incidence and prevalence by 2005, in 1991, the World Health Assembly set two TB control targets of a TB Case Detection Rate (CDR) of $70 \%$ and a TB treatment success rate of $85 \%$ [4]. In 2014, the World Health Assembly set a new strategy to end TB (defined as a TB incidence to $<10$ per 100,000 populations) by 2035 . A milestone in this strategy (the Global Plan Targets 2015 to 2025) was to reach $90 \%$ of all undiagnosed TB cases, $90 \%$ of all key populations, and attain at least $90 \% \mathrm{~TB}$ treatment success. Key populations, who are the most vulnerable and underserved 'at risk' populations include children, People Living with HIV (PLHIV), indigenous peoples, healthcare workers, mobile population, People who Inject Drugs (PWID), rural populations, urban populations, miners, prisoners, etc. Each government would define its key populations, plan and implement appropriate services to suit them, and measure progress towards these targets [5]. Due to its potential benefits, TB active case finding is recommended in regions with high TB prevalence, low detection rates and moderate to high treatment completion rates[6].

Contact investigation aims to identify and treat new cases of $\mathrm{TB}$, administer preventative therapy to contacts in whom TB is ruled out, or to closely monitor household transmission [7]. The likelihood of developing TB infection following a positive tuberculin skin test higher in younger persons [7]. Screening contacts of known TB cases is only useful if TB prevention Isoniazid Preventive Therapy (IPT) is given to persons with to prevent disease progression or TB treatment is effected [8]. Household contacts are the focus of TB contact investigation as they are at an increased risk of TB infection due to prolonged exposure with a TB index case [9]. Among household contacts, the risk of TB infection following exposure is highest among children [10]. In India, the yield of TB contact investigation among young children living in households of smear-positive TB cases ranged from 22-34\% and was higher (27-62\%) among children aged less than 10 years. The majority of TB infections among children aged $<3$ years occurred from household contact. Conducting household contact investigation in this age group, therefore, led to earlier diagnosis and treatment, prevent the occurrence of complications, and reduce the pool of future latent Tuberculosis infection (LTBI) cases [11]. In a study in Peru, the prevalence of TB detected in household contacts through active case finding was higher than through passive case finding $(0.91 \%$ vs. $0.18 \%)$. Similar results were observed among neighbors of the index case i.e. $0.22 \%$ vs. $0.08 \%$ respectively [12].

Ayles et al (2013) assessed the effect of two community-level interventions on TB control among adults aged 18 years and older. These interventions were implemented as follows; within the clinic alone (Group 1), within the clinic plus enhanced community level case-finding ECF (Group 2), enhanced community level case-finding plus household contact investigation (Group 3), and clinic plus enhanced community level case-finding plus household contact investigation (Group 4). The prevalence of TB was 832 per 100,000 populations. The adjusted prevalence ratio for ECF (Groups 2, 3 \& 4) vs. non-ECF (Group 1) was 1.09 and for household vs. non-household was 0.08 . The adjusted incidence ratio for a group of children followed up for 3 years for ECF vs. non-ECF was 1.36 and for household vs. non-household was 0.45 . As these interventions were designed to decrease TB transmission and ultimately TB prevalence, a longer follow-up period may have been required to assess their effect. Additionally, screening limited to only sputum smear-positive cases [13]. In India, among child contacts aged less than 5 years, $30 \%$ of TB cases would have been missed by focusing only on sputum positive index cases. This is because the majority of sputum negative cases do not have the minimum 10,000 (Acid-fast bacilli) AFB per ml required for a positive Zeihl-Neelsen stain [14]. 
In 2012, the World Health Organization (WHO) provided recommendations for investigating contacts of persons with infectious TB in low- and middle-income countries. However, these did not specify guidelines for specific programmatic conditions under which contact investigation should be conducted, the types of index cases to be prioritized for screening, specific protocols to be followed, or the contacts to be investigated (other than children aged $<5$ years and PLHIV) [15].

The TB program in Kenya was formally launched in 1980. The TB Case Notification Rate (CNR) in 1980 was 60 per 100,000 populations. Before the advent of HIV in Kenya, the TB CNR was falling at a rate of $4 \%$ per year. With the onset of the HIV epidemic in the 1990s, the TB CNR has begun increasing at $15 \%$ per year with a peak CNR of 300 cases per 100,000 population in 2005 [16]. In Kenya, between 1987 and 2006, the TB burden increased ten-fold due to Human immune-deficiency Virus (HIV) infection [17]. In 2016, the TB CNR in Kenya was 170 per 100,000 populations and in Kisumu County, 228 per 100,000 populations. Over 90\% $(75,986)$ of the TB case burden in Kenya was borne by adults. Kisumu County, one of the 47 counties in Kenya, contributed to 2,564 (3.4\%) of the 75,896 TB cases notified in 2016 [18]. In 2012, Kisumu County had the highest HIV prevalence in the country, $15.1 \%$ against the country's average of 5.6\% [19]. By 2005, Kenya had already reached the targets for TB Case Detection Rate (CDR) of 70\% and cure rates of 85\% [16]. From 2010, the TB program in Kenya increased expanded its focus on active case finding to screening HIV infected persons, new inmates in prisons, household or community cough monitors and household contacts of index cases of TB [20].

The Kenya TB program guidelines recommend IPT for TB prevention for all children aged $<5$ years who have been in household contact with a TB index case in whom a TB diagnosis has been excluded, as part of its underserved populations [21]. Although household contact investigation is included in Kenya TB Control policies, it has not been conducted in Kenya as it is the resource-intensive case [22]. TB screening among household contacts in Kenya is non-standardized and limited in scope to contact the invitation. Subsequently, the information on the contribution of contact investigation to early case detection in children is scarce or non-standardized. This precludes the assessment of its impact on TB control (WHO, 2012). The absence of information on the uptake of IPT uptake following among child contacts household with exposure further hampers such assessments [23]. In view of this discrepancy, there is a need to assess the contribution of contact investigation to diagnosing and preventing TB in children. The objective of this study was to compare the value of TB contact investigation to TB contact invitation in childhood TB control.

\section{Methods}

\subsection{Study design}

The TB contact investigation study was nested within a larger TB case detection study, a clusterrandomized trial conducted within 18 distinct geographical regions (clusters) in Kisumu County to determine the increase in TB case detection (for both drug-resistant and drug-susceptible TB) achieved through 3 different TB case detection strategies. In this study, a cluster was defined as a contiguous geographic unit with a population of approximately 25,000 persons based on the Kenyan 2009 census [24]. The TB case detection study's sites were selected based on being in a country with a national TB incidence rate $>50 / 100,000$ population, an in-country CDC office affiliated with existing TB activities which could easily be expanded to include contact investigation, a strong track record for successful collaboration with CDC, and either high HIV prevalence or high MDR-TB rates or both.

Cluster randomized trials provide evidence for the effectiveness of TB case detection in comparison 
to passive case finding [25] and should be used to provide evidence to support decision making [13]. A sub-group analyses of individuals randomized to the contact investigation strategy was compared to those that offered a standard of care. The CONSORT guidelines for reporting clinical trials with extensions for cluster trials were used to guide the preparation of this section [26].

\subsection{Study setting}

The study was conducted at 4 of the 7 subdivisions (which now approximate the new sub-counties) of Kisumu county [27]. ( 3 urban and 1 rural) and within 85 of its 204 health facilities that include 146 level II, 46 levels III, 21 level IV and 1 level V health facilities. There are 6 levels of health care facilities in Kenya; community, dispensaries, health centers, district hospitals, provincial hospitals, and national referral hospitals referred to as levels $1,2,3,4,5$, and 6 respectively [28]. The rural region was Kombewa and urban regions were Kisumu East, West, and Central. The regions were selected based on having 6 contiguous locations that contained approximately 25,000 persons based on the 2009 Census. The urban regions contained 12 units while the rural unit contained 6 units [29, 30]. Kisumu County located in Western Kenya, is a high TB burden, densely-populated region; it has a population of $1,097,307$ of whom $17.4 \%$ are aged less than 5 years. Its population density of 464.5 per square kilometer [29]. In 2016, the TB CDR was 228 against a national average of 170 per 100,000 populations [18]. Figure 4 shows health facility distribution in Kisumu sub-counties with different counties represented using different colors.

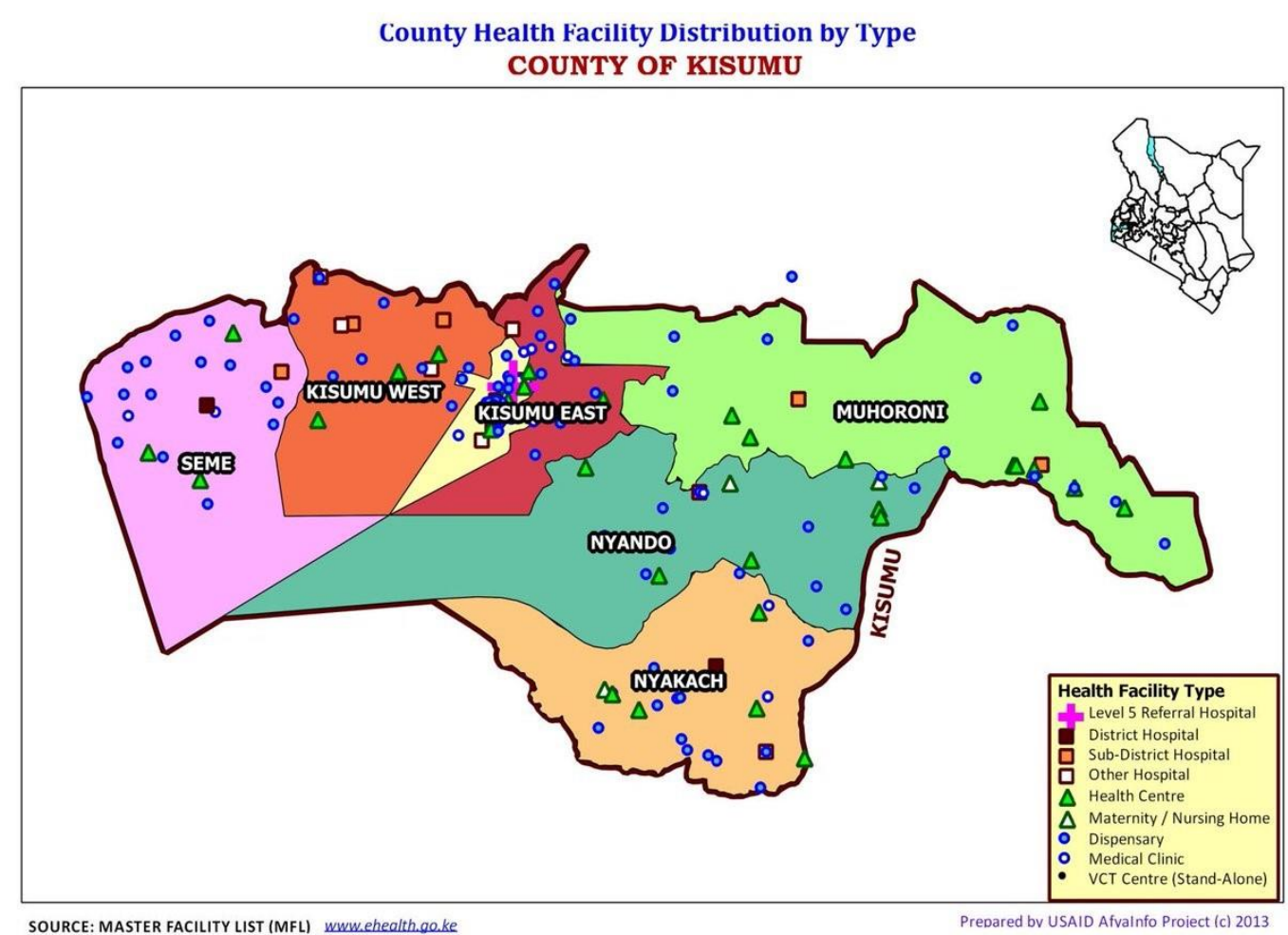

Figure 1: Kisumu sub-counties showing facility distribution (Source: Ministry of Health, 2017)

\subsection{Study population}

Health facilities within a location were assigned to the cluster to which the location found. The study population was comprised of all children diagnosed with TB or who received IPT within the 
specified health facilities randomized to a specific strategy.

To address this objective, the study population was comprised of all children aged less than 5 years who were diagnosed with TB and who received IPT at health facilities randomized to the TB contact investigation strategy and the standard approach (a.k.a. the contact invitation strategy) in the two years preceding the study period i.e. 2012 and 2013 (the pre-intervention period) and in during the two years that were designated the 'study period' (or intervention period i.e. 2014-2015).

The study population was chosen as it represents what would constitute TB control i.e. the number of TB cases diagnosed as a proxy for a decrease in morbidity and mortality from TB, and the number of children who received IPT as a proxy for the number of TB cases of TB prevented and therefore a decrease in TB incidence and prevalence [3]. The health facilities randomized to a specific control strategy would provide both a suitable intervention arm (e.g. the contact investigation strategy) and a suitable control arm the standard approach) because of randomization balances both the known and unknown confounding factors that would influence the outcome [31].

All persons residing within the region randomized to a TB case detection strategy or presenting to a health facility within the region were eligible for inclusion in the strategy to which that unit was randomized. This is because patients can present to a variety of health facilities for TB diagnoses within a larger area. As large areas were randomized to either receive or not receive an intervention, the impact of patients who went outside this area for TB diagnoses and treatment would be limited [13]. Before and after comparisons enabled the study to assess the presence of external changes that may have occurred in both the intervention and control arms during that may have contributed to changes in the outcome e.g. the introduction of new policies or practices in the TB program [32].

\subsection{Sample size and sampling procedure}

The sample population consisted of at least 19 children aged less than 5 years diagnosed with TB and 15 children aged less than 5 years who received IPT within the intervention and control arms. The study population of child contacts diagnosed with TB was computed as follows: To detect a $50 \%$ increase in the number of TB cases between pre and post-intervention period, with one-sided 5\% significance and a power of $80 \%$ power if any single intervention were anticipated to achieve its target [33].

$\left.\mathrm{n}=\left(\mathrm{p}_{1}\left(1-\mathrm{p}_{1}\right)+\mathrm{p}_{2}\left(1-\mathrm{p}_{2}\right)\right) /\left(\mathrm{p}_{1}-\mathrm{p}_{2}\right)^{2 *} . \mathrm{f}(\dot{\alpha}, \beta)\right)$

Where $\mathrm{p}_{1}$ is the proportion after the intervention and $\mathrm{p}_{2}$ is the proportion before the intervention and $\mathrm{f}$ $(\alpha, \beta)$ is a constant value for the power and significance level which at $80 \%$ power and $95 \%$ significance level is 7.9 [33].

TB Patient diagnostic rate $=\mathrm{TB}$ case detection rate $/ \mathrm{TB}$ prevalence was used as the proportion before the intervention [34]. In 2012, the TB case detection rate was 242 per 100,0000 [17], TB prevalence from the TB prevalence survey in 2015 was 586 per 100,000 [35]. Patient diagnostic rate (p1) was therefore 0.41 and $\mathrm{p} 20.82$.

$\mathrm{n}=\left((0.82(1-0.0 .82))+(0.41(1-0.41)) /(0.82-0.41)^{2} * 7.9\right.$

$\mathrm{n}=(0.82 * 0.18+0.41 * 0.59) / 0.41^{2} * 7.9$

$\mathrm{n}=18.3 \mathrm{n}=19$

A minimum sample size of $18 \mathrm{~TB}$ cases in each group (the pre and post intervention groups) would therefore be required to be $80 \%$ sure of being able to detect an increase in screening rates at the $5 \%$ significance level. The number of child contacts who received IPT was based on the WHO and Kenyan TB treatment recommendation of at least one child contact aged less than 5 years initiated on IPT for 
every index case of TB (1:1) [15].

The study employed a convenience sampling technique that collects information based on the availability and willingness of participants. This method is useful as participants were recruited from participating clinics prospectively and there were constraints on time and costs of conducting the study [36]. This method was also chosen due to a small target population as childhood TB represents less than $10 \%$ of all TB cases diagnosed [37], and as IPT implementation had just commenced, there was no existing information on which to base sample estimates (Tuberculosis, Leprosy, and Lung Diseases Coordinator, Nyanza Province, Personal Communication, $30^{\text {th }}$ August 2012).

\subsection{Randomization}

Four regions (two urban and two rural) were initially selected for the larger case detection study. The rural regions were Siaya and Kombewa Health Demographic and Surveillance System Area (HDSA); the urban regions were Kisumu Town East and Kisumu Town West. Kombewa's HDSA is located within Kisumu County while Siaya's HDSA is located within Siaya County.

Siaya and Kombewa region is constructed within previously defined DSSs, and areas within it have been well characterized, and most residential compounds geocoded [38, 39]. Urban areas were selected from the most densely populated areas of Kisumu Township and residences of persons registered for TB after deployment of study interventions were later geocoded [29]. The Kaloleni sub-location was excluded from this study, as it lied between the 2 largest hospitals in the Kisumu area, the Jaramogi Oginga Odinga Teaching, and Referral Hospital (JOOTRH) and the Kisumu District General Hospital (KDH) which has been renamed Kisumu East County hospital [30]. Each region was subdivided into 6 units that represented contiguous locations with approximately 25,000 persons; there were a total of 24 units. Units were stratified into rural and urban and within each stratum, units were randomized to either implement or not to implement an intervention.

\subsection{Implementation}

The randomization sequence was generated by the study statistician based on these probabilities without the knowledge of the investigators prior to study implementation. A waiver of informed consent was sought from the Kenya Medical Research Institute because participation in this study, presented only a minimal risk for participants, would not alter rights of participants who would continue to receive services as per the existing policies and guidelines, would lead to timely diagnosis of TB as interventions implemented in the study would employ the use of more sensitive tests and, and it would be impractical to conduct with study with individual informed consent as the study aimed to compare the programmatic utility of different TB case detection strategies.

Among the 12 urban units, 6 were randomly assigned to have the 3 enhanced health-facility conditions (H, HC, HCM). In the 6 units with enhanced health-facility conditions, 2 were randomly assigned condition $\mathrm{H}, 2$ were assigned condition $\mathrm{HC}$, and 2 were assigned condition HCM. Among the remaining 6 rural units, 2 were randomly assigned condition S, 2 were assigned condition C, and 2 were assigned condition $\mathrm{M}$. The same allocation procedure was used to assign interventions to the 12 rural units. However, for this analysis, Siaya was excluded due to other on-going TB control activities in the region that limited the scope of the TB case detection strategy and leading to an imbalance of randomization of strategies for the rural units. 


\subsection{Intervention}

The TB case detection study utilized 3 different strategies to enhance TB case detection namely;

- Household contact investigation (C): all patients diagnosed with TB were asked to elaborate a list of household members who would all undergo evaluation for TB.

- Facility-based active case finding $(\mathrm{H})$ : this involved the identification of all patients who screened positive for TB symptoms from all outpatient and inpatient departments and screening them for TB using sputum smear and Cepheid gene Xpert.

- Community-based active case finding using Mobile units (M): a mobile field site was established to move around selected communities to assess patients for TB symptoms and collect sputum specimens. The unit located itself within a particular area within the community for two weeks at a time and rotated throughout the community to be within a $2 \mathrm{~km}$ radius of every person within the community.

- The standard approach (S): in this group, there was no intervention. The program continued to diagnose patients as per their usual practice which was primarily based on the Directly Observed Treatment Short Course DOTS system of the passive finding of patients and confirmation of TB based sputum smear microscopy. Zones in the Standard Approach i.e. Contact invitation provided suitable control sites to compare the TB contact investigation study.

The strategies were combined as follows: Standard of care $(\mathrm{S})$, Health facility screening $(\mathrm{H})$, Community based mobile screening units (M), Contact investigations (C), Contact investigations and Health facility screening (HC), and all three the three strategies (HCM).

\subsection{Outcomes}

This study will describe the value of a TB contact screening strategy (e.g. contact investigation or contact invitation) as for changes in TB control achieved by that specific contact screening strategy. Cost comparisons are only possible if the program is mature, economic costs are well quantified and comparisons can be made across groups [40]. The TB program in Kenya has provided clear guidelines for the screening of household contacts [41]. However, this is limited by the fact that contact invitation rather than contact investigation is practiced in Kenya. As contact investigation is yet to be standardized in Kenya, an economic comparison between the two strategies would not be done (Tuberculosis, Leprosy, and Lung Diseases Coordinator, Personal Communication, 30th August 2012).

This study adopted the WHO TB control targets to define TB control i.e. TB control was defined in terms of the number of TB cases diagnosed among children aged less than 5 years, and the number of child contacts who have been exposed to an infectious case of TB who receive IPT [42]. The TB treatment outcomes will not be assessed as no further intervention will be provided in either group (contact investigation or contact screening) outside that which is received during routine care by the national TB control program after TB diagnosis. Although the outcome of IPT use that ought to be assessed is a decrease in TB incidence among children who received IPT; this would require a longer duration of follow up and a comparator group to assess the risk reduction accorded by IPT [43].

The outcome was measured at cluster-level. This was the number of TB cases diagnosed and persons who received IPT at health facilities randomized to a specific TB case detection strategy as reported by the TB program in the pre- and post-intervention period. The outcome of interest was the comparison of both the number of TB cases diagnosed among children aged less than 5 years and, number of children aged less than 5 years who received IPT in (i) the pre-intervention 


\subsection{Data collection}

Electronic data was extracted from the Kisumu County TB program databases. The following variables were collected from each arm; the number of TB cases diagnosed, and the number of children put on IPT before and after the commencement of the study. This information was obtained from the TB program registers in the year preceding the study and during the conduct of the study.

\subsection{Data analysis}

\section{Definitions of parameters of interest}

The contact investigation strategy was comprised of either a contact investigation strategy in isolation (where the health facility only conducted contact investigation), or a contact investigation strategy in combination with other TB case detection strategies (where a health facility implemented contact investigation in combination with either enhanced facility case detection strategy, or community mobile units or both). The contact invitation strategy was represented by health facilities where the standard case approach was used. Health facilities that were randomized to other TB case detection strategies that did not include contact investigation were excluded from the analysis i.e. either mobile units or enhanced facility detection in isolation or in combination

A TB case was described as an individual aged less than 5 years entered in the TB program register between the $1^{\text {st }}$ of January 2012 and 31st of December 2013 for the pre-intervention year, and between $1^{\text {st }}$ of January 2014 to the $31^{\text {st }}$ of December 2015 for the intervention years. A child who received IPT was described as an individual aged less than 5 years entered in the IPT register between the $1^{\text {st }}$ of January 2012 and 31st of December 2013 for the pre-intervention year, and between $1^{\text {st }}$ of January 2014 to the $31^{\text {st }}$ of December 2015 for the intervention years.

The dependent variable was TB control as measured by the number of TB cases detected (as a proxy for a decrease in morbidity and mortality from TB), and the number of children who received IPT to prevent TB (as a proxy for a decrease in TB incidence and prevalence).

The independent variable was the TB contact screening strategy which was either contact investigation or contact invitation (a.k.a. the Standard approach).

The CONSORT flow diagram was used to illustrate the progress of randomized units from randomization, intervention, and analysis [26]. Health facilities randomized to a contact investigation strategy were compared to those randomized to the standard approach in terms number, age group and gender of TB cases diagnosed in the pre-intervention years, type and rural and urban distribution of health facilities to assess for balance of randomization between the groups and limit chances of systematic error, using chi-square statistics [44].

Since the population sizes and TB case rates in the randomized populations were intended to be the same, the total number of cases reported during the intervening years was compared to pre-intervention years. The change in detection in each arm was also compared between arms.

Fishers' exact test was used to compare the number of TB cases diagnosed in the pre-intervention year by the arm and the pre- and post-intervention years. Similarly, analyses were conducted for the number of children who received IPT [45]. 


\section{Results}

\subsection{Results of randomization of health facilities}

Of 86 facilities identified for the study, 65 were randomized to a contact screening strategy. The majority (54) were randomized to a TB contact investigation strategy in isolation $(n=4)$, or in combination with health facility screening $(n=19)$, or in combination with both enhanced facility screening and mobile units $(n=31)$ with the remainder, $(n=11)$ randomized to the standard approach i.e. TB contact invitation. Clinics that were randomized to other strategies (e.g. mobile units $(n=6)$ or enhanced health facility screening $(n=14)$ in isolation or combination were excluded from the analysis (Figure 2)

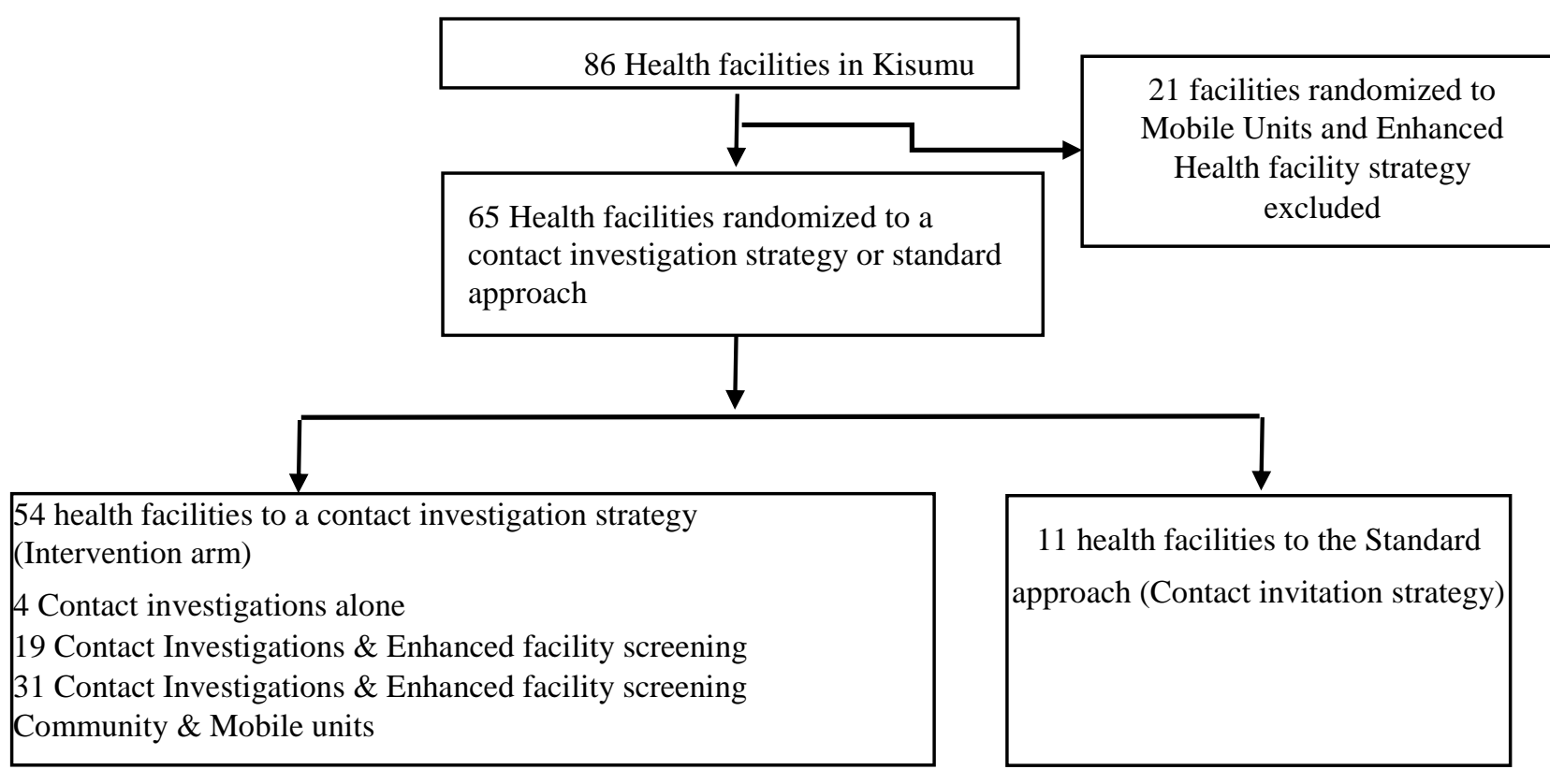

Figure 2: Randomization of health facilities to different strategies

\subsection{Baseline characteristics of health facilities by strategy}

Of 65 health facilities included, the majority were of level 2 (69\%), located in urban areas (64\%) and had a majorly male (56\%) adult patients (91\%). No facility was randomized to the Standard approach in rural locations. Facilities distribution did not differ by category of services or patient type (Table 1)

Table 1: Health facilities distribution to TB case detection strategies ${ }^{¥}$

\begin{tabular}{|l|c|c|c|c|}
\hline Characteristics & $\begin{array}{c}\text { No. of facilities } \\
\text { Total } \\
65(100 \%) \\
\mathrm{n}(\%)\end{array}$ & $\begin{array}{c}\text { Contact investigation } \\
\begin{array}{c}54(83 \%) \\
\mathrm{n}(\%)\end{array}\end{array}$ & $\begin{array}{c}\text { Standard approach } \\
11(17 \%) \\
\mathrm{n}(\%)\end{array}$ & P-value \\
\hline Health Facility & & & & \\
\hline Facilities level & & & & \\
\hline 2 & $45(69)$ & $38(84)$ & $7(16)$ & 0.18 \\
\hline 3 & $16(25)$ & $14(88)$ & $2(12)$ & \\
\hline $4 \& 5^{*}$ & $4(16)$ & $2(50)$ & $2(50)$ & \\
\hline Distribution & & & & $0(0)$ \\
\hline Rural & $21(33)$ & $22(100)$ & & - \\
\hline
\end{tabular}




\begin{tabular}{|l|c|c|c|c|}
\hline Urban & $44(67)$ & $33(75)$ & $11(25)$ & $\mathrm{N}=834$ \\
\hline$T B$ cases in $2013 \& 2012$ & $\mathrm{~N}=2713$ & $\mathrm{~N}=1879$ & & \\
\hline Age groups & & & $33(35)$ & 0.36 \\
\hline$<5$ years & $95(4)$ & $62(65)$ & $43(36)$ & \\
\hline $5-14$ & $123(4)$ & $79(64)$ & $757(30)$ & \\
\hline $15+$ years & $2495(92)$ & $1738(70)$ & & \\
\hline Gender & & & $473(31)$ & 0.88 \\
\hline Male & $1546(57)$ & $1073(69)$ & $361(31)$ & \\
\hline Female & $1167(43)$ & $806(69)$ & & \\
\hline
\end{tabular}

¥ Column percentages used throughout the table

*Levels grouped to eliminate cells containing zero, contact investigation facilities have 1 level 4 and 0 levels 5 facilities, and Standard approach has 2 level 4 and 1 level 5 facility

\subsection{Assessment of TB control activities}

In the pre-intervention and post-intervention years

The number of TB cases increased by 20; the majority were from the contact investigation arm (15/20; $75 \%)$ while the minority were from the contact invitation arm $(5 / 20 ; 52 \%)$. This increase was not statistically significant.

A before and after comparison of IPT administration could not be done since IPT implementation at all health facilities was not initiated until 2014. (Table 2)

During study implementation by TB case detection strategy

Between 2014 and 2015, there was a decrease in TB cases by 17 (12 from the Standard approach and 5 from the contact investigation arm); but this was not statistically significant (Table 2).

During the intervening years, health facilities randomized to the intervention arm contributed to $100 \%$ and 75\% (49/65) of the children put on IPT in 2014 and 2015 (Table 2).

Table 2: TB control in children by TB case detection strategy

\begin{tabular}{|c|c|c|c|c|c|c|}
\hline \multirow{3}{*}{ The measure of TB control } & \multicolumn{4}{|c|}{ Strategy } & \multirow{3}{*}{$\underline{\text { Total }}$} & \multirow{3}{*}{$\underline{\text { P-value }}$} \\
\hline & \multicolumn{3}{|c|}{$\begin{array}{l}\text { Contact investigation in isolation or } \\
\text { combination with other strategies }\end{array}$} & \multirow[t]{2}{*}{ 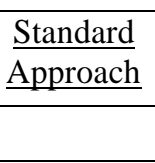 } & & \\
\hline & $\underline{\mathrm{C}}$ & $\mathrm{HC}+\mathrm{HCM}$ & Total & & & \\
\hline TB cases diagnosed & & & & & & $0.28 \dagger$ \\
\hline Pre-intervention years-Total & 8 & 54 & $62(65)$ & $33(35)$ & $95(100)$ & \\
\hline 2012 & 5 & 20 & 25 & 15 & 40 & \\
\hline 2013 & 3 & 34 & 37 & 18 & 55 & \\
\hline Post-Intervention Years-Total & 4 & 74 & $77(73)$ & $28(27)$ & $105(100)$ & $0.12 \dagger \dagger$ \\
\hline 2014 & 1 & 40 & 41 & 20 & 61 & \\
\hline 2015 & 3 & 33 & 36 & 8 & 44 & \\
\hline \multicolumn{7}{|l|}{ Children on IPT } \\
\hline Pre-intervention years*-Total & - & - & - & - & - & - \\
\hline 2012 & - & - & - & - & - & \\
\hline 2013 & - & - & - & - & - & \\
\hline Post-intervention years $* *$-Total & - & 49 & $49(75)$ & $16(25)$ & $65(100)$ & \\
\hline 2014 & - & 2 & $2(100)$ & - & $2(100)$ & - \\
\hline 2015 & - & 47 & $47(72)$ & $16(28)$ & $63(100)$ & \\
\hline
\end{tabular}


C-contact investigation, HC-contact investigation+ enhanced facility screening, HCM- contact investigation+ enhanced facility screening+ Community Mobile Units, S-Standard Approach (Contact Invitation)

$\dagger \mathrm{P}$ value comparing TB cases diagnosed in the pre and post-intervention period by the arm

$\dagger \uparrow \mathrm{p}$-value comparing TB cases diagnosed in the intervention period by the arm

*IPT implementation had not formally begun during the pre-intervention years

** Comparison by arm not done during intervention years as no children received IPT in 2014 in the standard arm

\section{Discussion}

We set out to compare the yield of TB contact investigation to contact invitation in childhood TB control. Data analysis and interpretation revealed three major findings. The number of children diagnosed with TB increased during the intervention years compared to the pre-intervention years. During the intervening years, the number of TB cases diagnosed at health facilities that were implementing a contact investigation strategy was higher than that at health facilities implementing a contact invitation strategy. In the intervention years, health facilities implementing a contact investigation strategy had a higher number of children that were put on IPT compared to facilities implementing a contact invitation strategy. Although a limited number of children were put on IPT in the first year of the post-intervention phase, this could be attributed to erratic IPT supply, and challenges in the documentation. (Personal communication, CTLC Coordinator, $10^{\text {th }}$ December 2018)

There was an increase in the number of TB cases and children put on IPT before and after the implementation of the study. In the literature, it has been established that Contact investigation leads to an increase in both the number of TB cases detected among children aged less than 5 years, as well as the number of children that were put on IPT [25]. Although all TB Case detection strategies have also been shown to increase TB case detection [13], cluster-randomized strategies have shown different yields between different TB case detection strategies with sometimes conflicting results. Over four years, the ZAMSTAR study showed a higher decrease in TB burden among persons randomized to a household strategy compared to those randomized to a community-level enhanced case-finding strategy where health education was given [13]. Contrary to this, the DETCT TB study in Zambia showed a higher yield among persons randomized to a mobile van unit compared to a household strategy. In the literature, mobile outreach services have been known to provide a higher yield. Although the authors state that the mobile unit may be associated with stigma since consultation occurs in front of others, the study did not investigate the preference for the mobile unit [46].

A Brazilian trial also showed a preference for a door-to-door strategy compared to a mobile van unit. The authors suggested that this could be because the effect of health education may take longer to motivate community members to change their health behavior [47]. Both studies proposed that household models led to personalized interaction, supported persons to assess their risks of infection and eased linkage to essential health services [13, 47]; these reasons apply to both household and enhanced facility screening strategies. However, such persons may have still presented to the clinic for screening in the absence of this study [12]. A combination of these reasons may have been responsible for the increase in TB CDR in the combined strategies. However, this study was unable to track participants over a long duration to assess the impact of IPT initiation. The study did also not enquire about the preference for a specific TB case detection strategy.

The results of this study are generalizable to other TB clinics in Kenya. The clinics randomized to the intervention arm did not differ from those that were randomized to the control arm [31]. The study employed a cluster randomized controlled trial which are powerful means of measuring the 
effectiveness of interventions; the level of evidence generated by randomized controlled trials is ranked higher than case-control or cohort studies [25]. The before and after comparison allowed for an assessment of the intervention over a longer period in which changes in the TB policies and guidelines would have impacted on the outcomes in both the intervention and control arms [32]. Although the difference in TB cases diagnosed did not achieve statistical significance, this was of clinical significance.

Our evaluation was not without limitations. Due to the limited numbers of children diagnosed with children within each specific strategy, we were unable to make a direct comparison of the yield of different TB case detection strategies when compared with the standard approach. Although the health facilities were not balanced in terms of rural and urban distribution, this was not a basis for comparison of study outcomes. Therefore, further analysis of the effect of balance was not conducted [44].

In conclusion, contact investigation provided the dual benefit of increasing TB case detection \& the opportunity to provide IPT and therefore impacting the burden of TB in childhood in Kisumu County. Timely TB diagnosis contributes to better treatment outcomes and therefore a decrease in morbidity and mortality from TB. TB prevention therapy contributes to a decrease in TB incidence and prevalence. Together, these two contribute to TB control in childhood.

We, therefore, recommend that TB programs implement TB Contact investigation among child household members that have been shown to enhance TB control activities in children in this region. Further research should be conducted to: Investigate acceptably and high-yield TB case-finding strategies.

Data availability: The number of Tuberculosis patients or TB exposed children on IPT data used to support the findings of this study are available from the corresponding author upon request.

Conflict of interest: None

\section{References}

[1] Porter, M.E., "What Is Value in Health Care?". New England Journal of Medicine, 363, $2477-$ 2481, 2010.

[2] Volpp, K., G. Loewenstein, and D. Asch, "Assessing value in health care programs". JAMA, 307, 2153-2154, 2012.

[3] Dowdle, W.R., "The principles of disease elimination and eradication". Bulletin of the World Health Organization, 76 Suppl 2, 22-25, 1998.

[4] Dye, C., M. Hosseini, and C. Watt, "Did we reach the 2005 targets for tuberculosis control?". Bull World Health Organ, 85, 364-9, 2007.

[5] World Health Organization Implementing the End TB Strategy: The essentials. https://www.who.int/tb/publications/2015/The Essentials to End TB/en/

[6] Murray, C.J.L. and J.A. Salomon, "Expanding the WHO tuberculosis control strategy: rethinking the role of active case-finding [The Pittsfield Lecture]". Int J Tuberc Lung Dis, 2, 9-15, 1998.

[7] Erkens, C.G., et al., "Tuberculosis contact investigation in low prevalence countries: a European consensus". Eur Respir J, 36, 925-49, 2010.

[8] MacIntyre, C.R. and A.J. Plant, "Impact of policy and practice on the effectiveness of contact screening for tuberculosis". Prev Med, 27, 830-7, 1998.

[9] Guwatudde, D., et al., "Tuberculosis in household contacts of infectious cases in Kampala, Uganda". Am J Epidemiol, 158, 887-98, 2003. 
[10] Sloot, R., et al., "Risk of Tuberculosis after Recent Exposure. A 10-Year Follow-up Study of Contacts in Amsterdam". American Journal of Respiratory and Critical Care Medicine, 190, 1044-1052, 2014.

[11] Banu Rekha, V.V., et al., "Contact screening and chemoprophylaxis in India's Revised Tuberculosis Control Programme: a situational analysis". Int J Tuberc Lung Dis, 13, 1507-12, 2009.

[12] Becerra, M.C., et al., "Expanding tuberculosis case detection by screening household contacts". Public Health Rep, 120, 271-7, 2005.

[13] Ayles, H., et al., "Effect of household and community interventions on the burden of tuberculosis in southern Africa: the ZAMSTAR community-randomized trial". Lancet, 382, 1183-94, 2013.

[14] Singh, M., et al., "Prevalence and risk factors for transmission of infection among children in household contact with adults having pulmonary tuberculosis". Arch Dis Child, 90, 624-8, 2005.

[15] World Health Organization Recommendations for investigating contacts of persons with infectious Tuberculosis in low and middle-income countries. http://apps.who.int/iris/bitstream/10665/77741/1/9789241504492_eng.pdf?ua=1

[16] World Health Organization A brief history of Tuberculosis in Kenya. https://www.who.int/tb/publications/tb-kenya-report/en/

[17] Division of Leprosy Tuberculosis and Lung Diseases Program Annual Report 2012. https://www.nltp.co.ke/annual-reports/\#

[18] National Tuberculosis Leprosy and Lung Disease Program Annual Report 2016. https://www.nltp.co.ke/annual-reports/

[19] National AIDS and STI Control Program NASCOP Kenya AIDS Indicator Survey 2012: Final Report. http://nacc.or.ke/wp-content/uploads/2015/10/KAIS-2012.pdf

[20] Division of Leprosy Tuberculosis and Lung Diseases Program Annual Report 2010. https://www.nltp.co.ke/annual-reports/

[21] Division of Leprosy Tuberculosis and Lung Diseases Program Guidelines for Management of Tuberculosis and Leprosy in Kenya. http://guidelines.health.go.ke:8000/media/TB_Treatment_GUIDELINES_2013.pdf

[22] Chakhaia, T., et al., "High Utility of Contact Investigation for Latent and Active Tuberculosis Case Detection among the Contacts: A Retrospective Cohort Study in Tbilisi, Georgia, 2010 2011". PLOS ONE, 9, e111773.2014.

[23] Chafe, R., "The Value of Qualitative Description in Health Services and Policy ResearchValeur de la description qualitative dans la recherche sur les politiques et services de santé". Healthcare policy $=$ Politiques de sante, 12, 12-18, 2017.

[24] Kenya National Bureau of Statistics 2009 Population and Housing Census. https://www.knbs.or.ke/publications/

[25] Fox, G.J., et al., "Contact investigation for tuberculosis: a systematic review and meta-analysis". Eur Respir J, 41, 140-56, 2013.

[26] Schulz, K.F., et al., "CONSORT 2010 statement: updated guidelines for reporting parallel group randomized trials". Int J Surg, 9, 672-7, 2011.

[27] Kenya Law Constitution of Kenya. www.kenyalaw/org/lex/act view.xql?actid=Const2010

[28] Ministry of Health Kenya Health Sector Strategic Investment Plan (KHSSPI) July 2013-June 2017. http://e-cavi.com/wp-content/uploads/2014/11/kenya-health-sector-strategic-investimentplan-2013-to-2017.pdf 
[29] County Government of Kisumu Kisumu County: First County Integrated Development Plan 2013-2017. https://roggkenya.org/wp-content/uploads/Kisumu_CIDP_2018-2022_CountyIntegrated-Development-Plan.pdf

[30] Ministry of Health Kenya Master Facility List. http://kmhfl.health.go.ke/

[31] Bruce, N.G., C. Pope, and D. Stanistreet, Quantitative methods for health research: A practical interactive guide to epidemiology and statistics. John Wiley Chichester. 2008

[32] Younge, J.O., et al., "Randomized study designs for lifestyle interventions: a tutorial". International Journal of Epidemiology, 44, 2006-2019, 2015.

[33] Whitley, E. and J. Ball, "Statistics review 4: sample size calculations". Crit Care, 6, 335-41, 2002.

[34] Borgdorff, M.W., "New measurable indicator for tuberculosis case detection". Emerg Infect Dis, 10, 1523-8, 2004.

[35] National Tuberculosis Leprosy and Lung Disease Program Kenya Tuberculosis Prevalence Survey 2016. https://www.chskenya.org/wp-content/uploads/2018/04/Final-TB-PrevalenceSurvey-Report.pdf

[36] Speak, A., et al., "Comparing convenience and probability sampling for urban ecology applications". Journal of Applied Ecology, 55, 2332-2342, 2018.

[37] World Health Organization Road Map for Childhood Tuberculosis. http://apps.who.int/iris/bitstream/10665/89506/1/9789241506137_eng.pdf?ua=1\&ua=1

[38] Sifuna, P., et al., "Health \& demographic surveillance system profile: The Kombewa health and demographic surveillance system (Kombewa HDSS)". Int J Epidemiol, 43, 1097-104, 2014.

[39] Odhiambo, F.O., et al., "Profile: the KEMRI/CDC Health and Demographic Surveillance System--Western Kenya". Int J Epidemiol, 41, 977-87, 2012.

[40] Issel, L.M., Health program planning, and evaluation: a practical, systematic approach for community health. Jones and Bartlett Publishers Sudbury, Mass. ; London. 2004

[41] Division of Leprosy Tuberculosis and Lung Diseases Program, National Paediatric Tuberculosis Care Guidelines. Division of Leprosy, Tuberculosis, and Lung Diseases Nairobi Kenya. 2011

[42] Azman, A.S., J.E. Golub, and D.W. Dowdy, "How much is tuberculosis screening worth? Estimating the value of active case finding for tuberculosis in South Africa, China, and India". BMC Med, 12, 216, 2014.

[43] Golub, J.E., et al., "Long-term protection from isoniazid preventive therapy for tuberculosis in HIV-infected patients in a medium-burden tuberculosis setting: the TB/HIV in Rio (THRio) study". Clin Infect Dis, 60, 639-45, 2015.

[44] Festic, E., B. Rawal, and O. Gajic, "How to improve assessment of balance in baseline characteristics of clinical trial participants-example from PROSEVA trial data?". Annals of translational medicine, 4, 79-79, 2016.

[45] Parab, S. and S. Bhalerao, "Choosing statistical test". International Journal of Ayurveda research, 1, 187-191, 2010.

[46] Corbett, E.L., et al., "Comparison of two active case-finding strategies for community-based diagnosis of symptomatic smear-positive tuberculosis and control of infectious tuberculosis in Harare, Zimbabwe (DETECTB): a cluster-randomized trial". Lancet, 376, 1244-53, 2010.

[47] Miller, A.C., et al., "Controlled trial of active tuberculosis case finding in a Brazilian favela". Int J Tuberc Lung Dis, 14, 720-6, 2010. 